indicated.

Registry No. PABA, 150-13-0; carbaryl, 63-25-2; 1-naphthol, 90-15-3.

\section{LITERATURE CITED}

(1) Kuhr, R. J.i Dorough, H. W. Carbamate Insecticides: Chemistry, Blochemistry and Toxicology; CRC Press: Cleveland, $\mathrm{OH}, 1976$.

(2) Bowman, M. C.; Beroza, M. Residue Rev. 1967, 17, 23-24.

(3) Argauer, R. J.; Shimanuki, H.; Alvarez, C. C. J. Agric. Food Chem $1970,18,688-691$

(4) Argauer, R. J.; Bontoyan, W. J. Assoc. Off. Anal. Chem. 1970, 53 1166-1169.

(5) Ott, D. E.; Ittig, M.; Friestad, H. O. J. Assoc. Off. Anal. Chem. 1971, $54,160-164$.

(6) Argauer, R. J.; Webb, R. E. J. Agric. Food Chem. 1972, 20, 732-734.

(7) Larkin, M. J.; Day, M. J. Anal. Chim. Acta 1979, 108, 425-427.

(8) Aaron, J. J.; Some, N. Analusis 1982, 10, 481-485.

(9) Roman, M.; Gutierrez, F. A.; Mahedero, M. C. Atinidad 1983, 40 369-372.

(10) Moye, H. A.; Winefordner, J. D. J. Agric. Food. Chem. 1965, 13, 516-518.

(11) Kirkbright, G. F.; Shaw, E. S. Can J. Spectrosc. 1983, 28, 100-103

(12) Vanelli, J. J.; Schulman, E. M. Anal. Chem. 1984, 56, 1030-1033

(13) Su, S. Y.; Asafu-Adjaye, E. B.; Ocak, S. Analyst (London) 1984, 109 1019-1023.

(14) Vo-Dinh, T.; Winefordner, J. D. Appl. Spectrosc. Rev. 1977, 261-294.

(15) Parker, R. T.; Freedlander, R. S.; Dunlap, R. B. Anal. Chim. Acta 1980, 119, 189-209.

(16) Hurtublse, R. J. Solld Surface Luminescence Analysis, Theory, Instrumentation, Appllcations: Marcel Dekker: New York, 1981.

(17) Vo-Dinh, T. Room Temperature Phosphorimetry for Chemical Analysis; Wiley: New York, 1984.

(18) Paynter, R. A.; Wellons, S. L.; Winefordner, J. D. Anal. Chem. 1974, 46, 736-739.

(19) Bateh, R. P.: Winefordner, J. D. Talanta 1982, 29, 713-717.

(20) Su, S. Y.; Bolton, D. L.; Winefordner, J. D. Chem. Blomed. Environ. Instrum. 1982, 12, 55-64.

(21) McAleese, D. L.; Dunlap, R. B. Anal. Chem. 1984, 56, 600-601.
(22) Campliglla, A. D. MSc. Thesis, University of Brasilia, 1985.

(23) Aldrich Catalog Handbook of Fine Chemicals; Aldrich Chemical Co.: Milwaukee, WI 1984.

(24) Kaneco, H.; Hosoya, S.; Liyama, K.; Nakano, J. J. Wood Chem. Technol. 1883, 3, 399-411.

(25) Weichelt, Th.; Zlechmann, W. Naturwissenschaften 1977, 64 , 638-639.

(26) Gellestedt, G.; Petterson, E.-L. Acta Chem. Scand., Ser. B 1975 B29, 1005-1010

(27) De Lima, C. G.; de M. Nicola, E. M. Anal. Chem. 1978, 50, $1658-1665$

(28) Andino, M. M.; Kosinski, M. A.; Winefordner, J. D. Anal. Chem. 1986 $58,1730-1733$.

(29) De Lima, C. G.; Andino, M. M.; Winefordner, J. D. Anal. Chem. 1986, $58,2867-2869$.

(30) Long, G. L.; Winefordner, J. D. Anal. Chem. 1983, 55, 712A-724A.

(31) Atomic Spectrometry Short Course; Department of Chemistry, Loughborough Unlversity of Technology: Loughborough, 1982.

'Present address: Department of Chemistry, University of Florida, Gainesville, FL 32611.

Departamento de Quimica

Andrês D. Campiglia ${ }^{1}$ Clausius G. de Lima*

Universidade de Brasilia

Brasilia, Distrito Federal 70910, Brazil

RECEIVED for review November 12, 1986. Resubmitted May 11, 1987. Accepted August 3, 1987. These investigations were supported by the University of Brasilia and a FINEP (Brazilian Studies and Projects Financing Agency) grant, which allowed us to buy the spectrofluorometer used. Abstracted in part from the MSc thesis submitted by Andrês D. Campiglia to the Departamento de Quimica, Universidade de Brasilia, as a partial requirement for the degree.

\title{
Nature of Anionic Sites in Plasticized Poly(vinyl chloride) Membranes
}

Sir: The working mechanism of neutral-carrier-solvent polymeric membranes is at present still not fully understood. One of the unresolved problems is the origin of the cation permselectivity that is exhibited by commonly used plasticized poly(vinyl chloride) membranes (PVC membranes) (1). This behavior is essential for proper functioning of neutral-carrier membranes, as was already recognized by Perry et al. (2) in 1976. They demonstrated that cellulose acetate membranes loaded with valinomycin only show potassium vs sodium selectivity if cation permselectivity is already present. The generally accepted explanation for this permselectivity is the presence of anionic sites embedded in the membrane. Perry et al. fabricated membranes containing such sites by mixing cellulose acetate with variable amounts of cellulose acetate modified with sulfonic acid groups. However, hitherto the exact nature and origin of anionic sites in PVC, commonly used for the preparation of ion-selective membranes, has not been established.

Thoma et al. (3) proposed a mechanism involving small water clusters, present in the membrane, which contain $\mathrm{OH}^{-}$ ions. If this were the case, the bulk conductivity of the membrane would grow with increasing soaking time, a phenomenon that was very recently contradicted by experiments of Horvai et al. (4). Conductivity experiments described in the same paper also exclude the possibility that the anionic sites originate from the tetrahydrofuran (THF) solvent or the plasticizer.

With these results, our efforts were focused on the identification of ionic components in the PVC polymer, using impedance measurements, X-ray fluorescence, secondary ion mass spectrometry (SIMS), and X-ray photoelectron spectroscopy (XPS). We assume that these components may arise from different origins: (1) polymer-bound initiating groups, e.g. $\mathrm{ROSO}_{3}^{-}$groups or $\mathrm{RSO}_{3}^{-}$groups generated by a metal persulfate anion radical initiator (5); (2) oxidation resulting in $\mathrm{RCO}_{2}{ }^{-}$groups (6); (3) remainders of surfactants used during emulsion or suspension polymerization, like alkyl sulfates or phosphates, or dialkyl sulfosuccinates (7). We have used various experimental techniques to disclose the type of the groups acting as anionic sites.

\section{EXPERIMENTAL SECTION}

Chemicals. Di-n-butyl phthalate (DBP) was purchased from BDH Chemicals, Ltd. THF was obtained from Janssen Chimica and was redistilled prior to use. High molecular weight PVC was obtained from Fluka (referred to as Fluka-PVC) and BASF (referred to as BASF-PVC). Carboxylated PVC (1.4 wt \% COOH) was purchased from Janssen Chimica (designated $\mathrm{COOH}-\mathrm{PVC}$ ). All other chemicals used were analytical grade reagents.

Apparatus. Frequency response analysis (FRA measurements) was performed with a Solartron 1170 frequency response analyzer. The applied sinusoidal voltage was $10.0 \mathrm{mV}$. Pt electrodes with an estimated area of $0.2 \mathrm{~cm}^{2}$ were used. It was confirmed that with the concentrations used, and in the frequency range $10^{4}-1$ $\mathrm{Hz}$, the electrode impedance could be neglected relative to the membrane impedance.

XPS measurements were carried out with an ESCALAB MK II (VG, Applications Laboratory) for the survey spectra and a XSAM 800 (Kratos, Applications Laboratory) for the detailed spectra. Note that only after sputtering $900 \AA$ from the surface 
Table I. Resistivity and Relative Permittivity of

Plasticized Fluka-, BASF-, and COOH-PVC Membranes

after $20 \mathrm{Min}$ of Contact with $10^{-3} \mathrm{M} \mathrm{KCl}$ Solutions

\begin{tabular}{lcr} 
& $10^{8} \rho_{\mathrm{b}}, \Omega \mathrm{cm}$ & $\epsilon_{\mathrm{r}}$ \\
Fluka & 21 & 7 \\
BASF & 22 & 7 \\
COOH & 5.5 & 10 \\
\hline
\end{tabular}

with argon, a sulfur signal was measured.

SIMS measurements were performed with an Ion Microprobe Mass Analyzer (IMMA) (Applied Research Laboratories). The investigated membrane area was $75 \times 75 \mu \mathrm{m}^{2} ; \mathrm{N}_{2}{ }^{+}$ions were applied as primary ions, at $20 \mathrm{keV}, 1.5 \mathrm{nA}$.

$\mathrm{X}$-ray fluorescence spectra were measured with a Philips PW 140 wavelength dispersive spectrometer. A PE crystal was used, and a slow scan was carried out in order to increase the signal to noise ratio.

Procedure. The plasticized PVC membranes were formed by spin-coating PVC-DBP-THF, $1 / 2 / 10$ (wt), solutions at $1000 \mathrm{rpm}$ on an appropriate substrate. This resulted in the following membrane thicknesses: BASF-PVC, $6 \mu \mathrm{m}$; COOH-PVC, $5 \mu \mathrm{m}$; Fluka-PVC, $4 \mu \mathrm{m}$. Thicknesses were determined by interferometry. Supported by a nylon mesh, the plasticized PVC-membranes were mounted on a plastic cylinder and sealed with a Viton O-ring. The active membrane area was $0.38 \mathrm{~cm}^{2}$. Samples for XPS and SIMS measurements were cut out of the substrates, and in the case of SIMS measurements a thin gold layer was evapo. rated on top to avoid too much electrostatic charging of the surface. The charging effect with XPS measurements was excluded by taking the $\mathrm{C}$ 1s peak as an internal standard at 285 $\mathrm{eV}$. From the XPS spectrum the ratio of the carbon and chlorine concentration was calculated to vary between $\mathrm{C}: \mathrm{Cl}=1.9: 1$ and $\mathrm{C:Cl}=2.1: 1$ (theoretical ratio, $2: 1$ ), for the different apparatuses used, which indicates the variation in the sensitivity coefficient with the experimental setup. In the other measurements (detailed XPS spectrum and SIMS spectra) too much uncertainty about the sensitivity factors prevented accurate calculations.

$\mathrm{X}$-ray fluorescence was measured on a pressed pellet made from Fluka-PVC powder without plasticizer. The $\mathrm{COOH}$ content of $\mathrm{COOH}-\mathrm{PVC}$ was determined by potentiometric titration.

\section{RESULTS AND DISCUSSION}

Impedance Measurements. We investigated the possibility that carboxyl groups, attached to the PVC, might provide the immobile anionic groups in PVC by comparing the bulk resistivity $\rho_{\mathrm{b}}(\Omega \mathrm{cm})$ of $\mathrm{COOH}-\mathrm{PVC}$ with that of high molecular weight PVC. The bulk resistivity was calculated from impedance plane plots; it is related to the membrane bulk resistance by $\rho_{\mathrm{b}}=(A / d) R_{\mathrm{b}}$, where $A$ is the membrane area $\left(\mathrm{cm}^{2}\right), d$ the membrane thickness $(\mathrm{cm})$, and $R_{\mathrm{b}}$ the measured resistance $(\Omega)$. In Figure 1 the normalized impedance plane plots are shown for the three polymers. From $\omega_{m}$ (the frequency at which the imaginary part of the impedance reaches its maximum), the relative permittivity $\epsilon_{\mathrm{r}}$ can be calculated via $\epsilon_{\mathrm{r}}=1 / \epsilon_{0} \omega_{\mathrm{m}} \rho_{\mathrm{b}}$. The results are summarized in Table I. From the results in Table I it can be concluded that the bulk resistivity of $\mathrm{COOH}-\mathrm{PVC}$ is about a factor of 3-4 smaller than that of Fluka- or BASF-PVC. If carboxyl groups in Fluka- or BASF-PVC are responsible for the observed conduction, then the resistivity in $\mathrm{COOH}-\mathrm{PVC}$ must be lower than that which is actually measured. The difference in resistivity experienced is probably due to the higher relative permittivity, which leads to a higher degree of ionization of present ion pairs. Possibly in COOH-PVC the carboxyl groups contribute to the observed conduction, but in Fluka- and BASF-PVC they certainly are not responsible for the measured resistivity. The gradual increase of $\rho_{b}$ with time observed with BASF-PVC and to a smaller extent with FlukaPVC, that was also experienced by Horvai et al. (4), leads to the conclusion that the ionic species are not completely immobile but slowly leach out of the membrane. Therefore, it

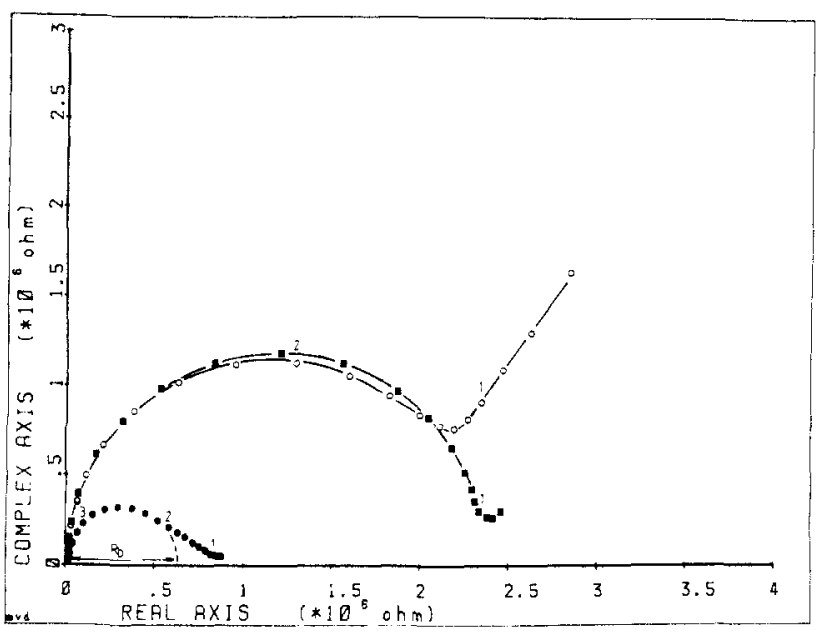

Figure 1. Impedance plane plots of Fluka- $(O)$, BASF- ( $\square$ ), and COOH-PVC (O) in $10^{-3} \mathrm{M} \mathrm{KCl}$ after $20 \mathrm{~min}$ (normalized to $d=4 \mu \mathrm{m}$ ). The figures on top of the curves indicate the logarithm of the measuring frequency.

is unlikely that these anionic groups are chemically bound to the polymer.

$\mathbf{X}$-ray Fluorescence. We investigated the presence of sulfur- or phosphorus-containing groups in Fluka-PVC with $\mathrm{X}$-ray fluorescence. In the spectrum a small but clear peak was noticeable at $\theta=76^{\circ}$, giving an indication for sulfur. Phosphorus was not found by this technique.

SIMS Measurements. We also investigated the FlukaPVC with SIMS. Both cationic and anionic depth profiles were recorded (Figure 2). From the results shown in Figure $2 \mathrm{a}$ it can be concluded that $\mathrm{Na}^{+}$and $\mathrm{K}^{+}$are initially present in the membrane, probably as counterions for the immobile anions. It should be noted that the amount of $\mathrm{Na}^{+}$and $\mathrm{K}^{+}$ is exaggerated since SIMS is very sensitive to metal cations. In the anion profile (Figure $2 \mathrm{~b}$ ) both $\mathrm{S}^{2-}$ and $\mathrm{O}^{2-}$ are present in an estimated atomic ratio of $\cong 1: 5-10$. No phosphorus was detected. Although the sulfur signal was small, it was significantly above the detection limit of the instrument.

XPS Measurements. In order to investigate the chemical state of the apparently present sulfur, we performed XPS measurements. In Figure 3 a survey spectrum of Fluka-PVC is shown. $\mathrm{C} 1 \mathrm{~s}, \mathrm{Cl} 2 \mathrm{~s}, \mathrm{Cl} 2 \mathrm{p}$, and $\mathrm{O}$ 1s peaks are displayed clearly, and the $\mathrm{C}: \mathrm{Cl}$ ratio was in agreement with the theoretical value. In the $S 2 p$ region a small signal was obtained only with increased sampling time and after sputtering (see Figure 4). The shape of the S $2 p$ peak and its position indicate that the sulfur is present in more than one oxidation state (8). Although some influence of the sputtering procedure on the chemical state of sulfur cannot be excluded, the measurements suggest in our opinion that a mixture of sulfate and sulfonate groups is present in the polymer.

\section{CONCLUSION}

From the combined results of FRA, X-ray fluorescence, SIMS, and XPS we conclude that in Fluka-PVC sulfur-containing components are probably present. These components might be sulfate or sulfonate groups and act as the anionic sites. FRA measurements indicate that these sites are presumably present in the form of emulsifier residues, and are not bound to the polymer matrix. The amount of anionic sites appears to be uncontrolled and depends probably on purification methods. In order to avoid this, and to prevent the possibility of leakage toward aqueous solution, it seems favorable to design systems in which anionic groups are solely introduced by attaching them to the polymer. This idea was already suggested and investigated by Cutler et al. (9) in 1977 for surfactant-sensitive membrane electrodes and proved to 

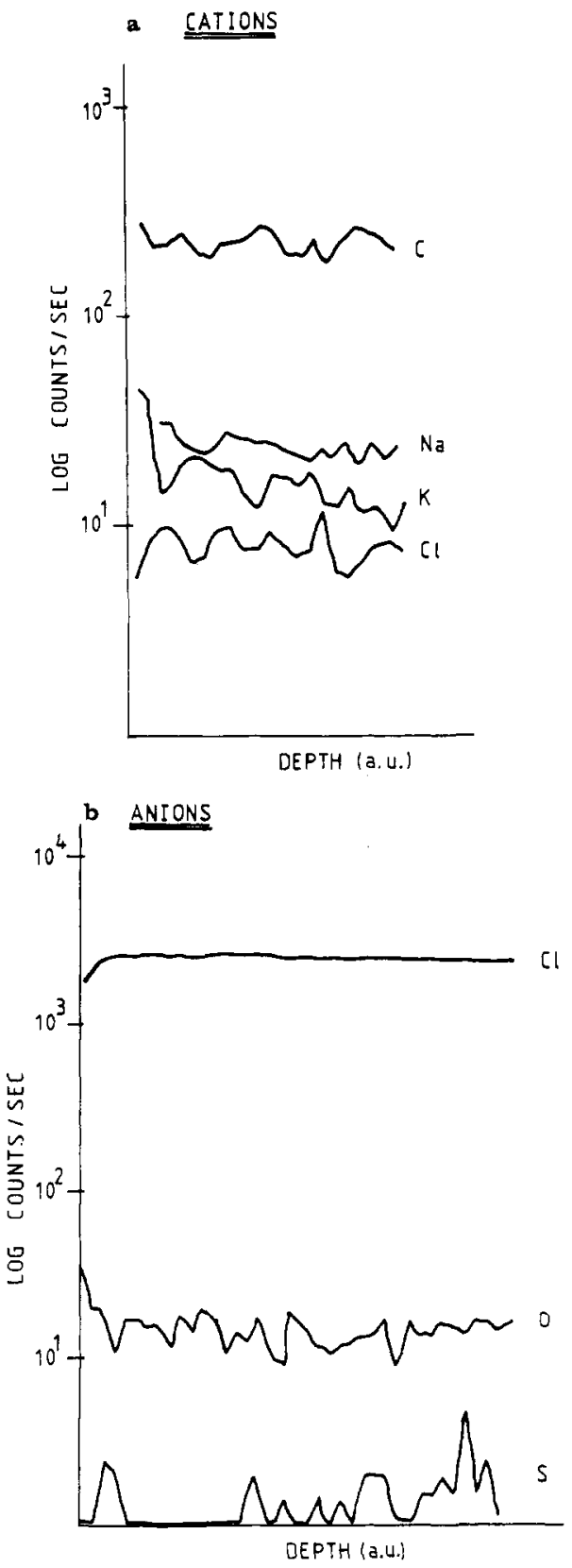

Figure 2. (a) Cation depth profile of Fluka-PVC. (b) Anion depth profile of Fluka-PVC.

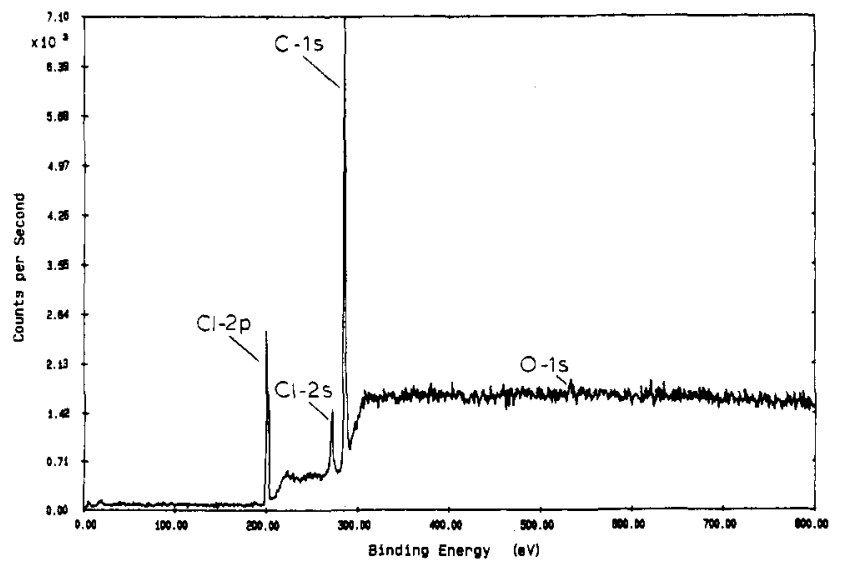

Figure 3. XPS survey spectrum of Fluka-PVC.

be rather successful. However, the role of water in the ionsensing mechanism should not be underestimated, for example, its influence on the relative permittivity or on the ionic dissociation. The relatively large water uptake observed with

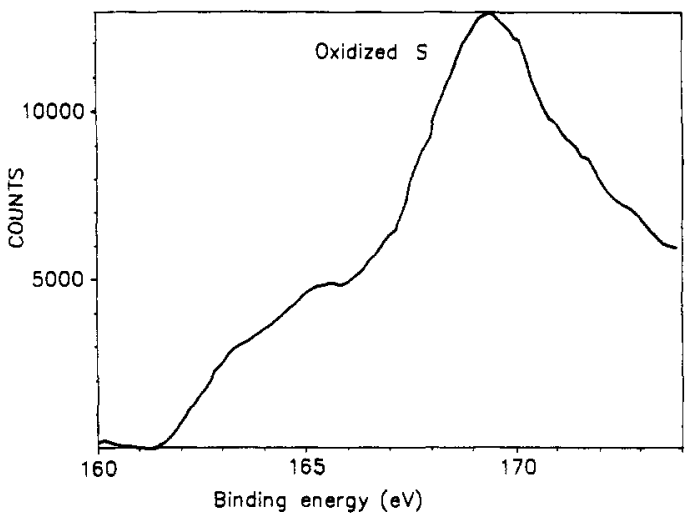

Figure 4. Detalled XPS spectrum of $S 2 p$ region (acquisition time 20 min; measured after sputtering 900 A).

plasticized PVC membranes (4) could well be caused by emulsifier residues. Their well-known ability to form reversed micelles (10) may contribute to the formation and stabilization of the water clusters (11). Further investigations are necessary to clarify whether or not emulsifiers are essential membrane components for proper functioning of ion-selective electrodes.

\section{ACKNOWLEDGMENT}

We thank Bernard Bouwkamp and Ico Vinke for use of the FRA experimental setup, D. J. Gras for performing SIMS measurements, and Rico Keim for interpretation of the XPS spectra.

Registry No. PVC, 9002-86-2.

\section{LITERATURE CITED}

(1) Morf, W. E.; Simon, W. Helv. Chim. Acta 1986, 69, 1120-1131.

(2) Perry, M.; Löbel, E.; Bloch, R. J. Membr. Sci. 1976, 1, 223-235.

(3) Thoma, A. P.; Viviani-Nauer, A.; Arvanitis, S.; Morf, W. E.; Simon, W. Anal. Chem. 1977, 49, 1567-1572.

(4) Horvai, G.; Grâf, E.; Tôth, K.; Pungor, E.; Buck, R. P. Anal. Chem. $1986,58,2735-2740$.

(5) O'Driscoll, K. F.; Ghosh, P. Structure and Mechanlsm in Vinyl Polymerization; Marcel Dekker: New York, 1969; Chapter 3.

(6) Jellinek, H. H. G. Degradation and Stabilization of Polymers; Elsevier: New York, 1983; Chapter 1.

(7) Van der Hoff, B. M. E. Solvent Properties of Surfactant Solutions ; Marcel Dekker:" New York, 1967; Vol. 2, Chapter 7.

(8) Biggs, D.: Seah, M. P. Practical Surface Analysis; Wiley: New York, 1985; Appendix 4.

(9) Cutler, S. G.; Meares, P.; Hall, D. G. J. Electroanal. Chem. 1977, 85 , 145-161.

(10) Tadros, Th. F. Surfactants; Academic: London, 1984.

(11) Brown, G. L. Water in Polymers; ACS Symposium Series; American Chemical Soclety: Washington DC, 1980; Chapter 26.

${ }^{1}$ Also Faculty of Electrical Engineering.

\section{Albert van den Berg ${ }^{1}$ \\ Peter D. van der Wal \\ Maria Skowrońska-Ptasińska \\ Ernst J. R. Sudhölter}

Faculty of Chemical Technology

University of Twente

7500 AE Enschede, The Netherlands

\section{Piet Bergveld}

Faculty of Electrical Engineering

University of Twente

7500 AE Enschede, The Netherlands

RECEIVED for review February 4, 1987. Accepted August 5, 1987. Financial support for this work was obtained from the Twente Centre of Microelectronics (CMET). 